\title{
Reflexiones Sobre el Aborto: Eficacia de las Normas que lo Regulan en Colombia
}

\section{Reflection on abortion: efficacy of the regulating norms In Colombia}

\author{
CLAUDIA PATRICIA DE LA ROSA GUZMÁN \\ Directora Programa de Derecho Facultad de Ciencias Jurídicas y Políticas \\ Universidad de San Buenaventura Bogotá. Correo electrónico: \\ cdelarosag72.abogada@gmail.com
}

Director of the law undergraduate degree in the Faculty of Juridical and Political Science Universidad de San Buenaventura, Bogota. E-mail: cdelarosag72.abogada@gmail.com

Fecha de recepción: 2 de septiembre de 2011 Fecha de evaluación: 15 de septiembre de 2011 Fecha de aprobación: 29 de noviembre de 2011

RESUMEN. En este articulo se pretende, en primer lugar, hacer claridad conceptual a juicio del autor sobre el aborto y su legalización, dar una mirada a la forma en que se ha tratado el problema en Colombia desde sus fuentes jurídicas, es decir, observar cual ha sido el recorrido jurídico, bien sea, por actos legislativos o a través de sentencias judiciales, que han contemplado el tema y por medio de los cuales ha cambiado en el tiempo su tratamiento de acuerdo a las diferentes circunstancias de cada época, sin que haya dejado nunca de 
establecerse como un delito. Por último, determinar qué criterios son aplicables cuando se trata de establecer un escenario de discusión en el que éstos, de acuerdo a lo preceptuado en la Constitución Política de 1991, sean los democráticamente pertinentes y apropiados para buscar una solución válida y eficaz dirigida a contrarrestar las consecuencias sociales que él problema representa.

PALABRAS CLAVE. Aborto, delito, sentencias, actos legislativos, eficacia normativa, escenarios de discusión, objeción de conciencia.

ABSTRACT. In this article is intended to, firstly, to make conceptual clarity in the author's opinion on abortion and its legalization, give a look at the way in which we have dealt with the problem in Colombia since their legal sources, i.e. observe which has been the legal trail, either, by legislative acts or through judicial rulings, which have been referred to the topic and through which has changed in time, their treatment, according to the different circumstances of each time, without that has never ceased to be established as a crime. Finally, to determine what criteria are applicable when it comes to establishing a scenario for discussion in which these, according to the provisions of the Constitution of 1991, the democratically relevant and appropriate to find a valid solution and effective addressed to counteract the social consequences that the problem represents. 
KEYWORDS. abortion, crime, sentence, legislative act, normative efficacy, discussion scenario, conscience objection

Tipo de Artículo: Reflexión Académica

\section{INTRODUCCIÓN.}

Del tema del aborto seguramente se ha dicho un poco de todo y hasta resultaría predecible, en apariencia, retomar su estudio; sin embargo, hacerlo ha constituido a título personal una intención constante por la relevancia del tema. En el transcurso de mi actividad como abogada y en el ejercicio de la cátedra universitaria, he podido observar que frente a esta situación, son muchas los debates, discusiones, posiciones y retos, pero pocas las posibles soluciones que determinen con precisión un criterio equilibrado y maximicen a su vez, la observancia ecuánime de aspectos como la vida, la muerte y al tiempo los derechos. Es un tema apasionante y al tiempo inagotable, precisamente por la naturaleza de los conflictos que genera sus contraposiciones.

Es un problema que nos hace entrar en un terreno de "arenas movedizas" en las que con toda seguridad por donde tratemos de indagar y establecer criterios, 
vamos a herir susceptibilidades de posiciones contrarias. Por eso es tan importante determinar el tipo de terreno sobre el cual se pueden debatir los puntos encontrados entre pro vidas y pro abortistas, desistiendo de una vez por todas intentar fallidamente discutir el tema en forma exclusiva desde el terreno de lo moral, porque aunque el problema tiene matices morales, nadie cede a sus principios.

La Conveniencia de trasladar la discusión desde el escenario donde ha estado por mucho tiempo, o sea, el moral o religioso, o bien desde el escenario actual, es decir, el jurídico, al escenario político, donde realmente debería hacerse el debate, es motivo de exposición en esta oportunidad.

Esta investigación es de tipo bibliográfico que acude a fuentes históricas y descripciones sociológicas; no obstante se requiere la verificación de datos, información, efectividad de las decisiones, eficacia de la normatividad, para efectos de lo cual se debe indagar con las entidades involucradas en el tema, las distintas posiciones al respecto y establecer la coherencia en la praxis respecto de la eficacia de las normas tanto legales como jurisprudenciales en el tema del aborto. El procedimiento que se sigue en cuanto a la metodología implica que inicialmente se haga una recopilación de información sin distingo de autores nacionales y extranjeros, jurisprudencia nacional de la Corte Constitucional y de la 
Corte Suprema de Justicia, doctrina nacional y extranjera, compilación de normas de orden legal, judicial y administrativas que den cuenta de la reglamentación sobre el aborto en Colombia.

\section{PRECISIÓN DE CONCEPTOS}

En el desarrollo de este tema tan controvertible, se ha hecho referencia casi siempre a la utilización de términos que, parece tuvieran una misma connotación, sin embargo, crean confusión al momento de iniciar el estudio del mismo, y vale la pena aclarar para estos efectos: Uno de los aspectos más relevantes en cuanto al aborto es la discusión que surge respecto de si debe legalizarse o no en Colombia, lo cual denota una inapropiada utilización de la terminología jurídica con la que se trata el tema, pues haciendo una interpretación de los términos de manera muy particular, el aborto está legalizado desde hace mucho tiempo, y no podemos establecer erradamente que no está legalizado por estar prohibido. Legalización, Permisibilidad, Prohibición y penalización son términos diferentes al momento de determinar cuál es la situación jurídica de este problema en nuestro país.

De acuerdo a las diferentes acepciones que tiene la palabra legalizar, entendemos 
básicamente por ésta, "Dar estado legal a algo", "otorgar carácter legal a algo" o "la concesión del estado legal a una cosa"; lo cual indica que cuando se habla de legalización estamos ante una situación en la que se busca que ciertos hechos sean regulados por el ordenamiento jurídico vigente, es decir, el derecho positivo, bien sea para permitir o prohibir la conducta y se establezca la penalización como consecuencia de su prohibición, como en el caso que nos ocupa.

En Colombia no podemos hablar de legalización, cuando lo que indica la historia jurídica de este problema es que el aborto se encuentra reglado o legalizado de tiempo atrás, atribuyéndole el tratamiento especifico del mismo, a la norma penal por su consecuente prohibición. En la actualidad el delito del aborto se encuentra regulado por el Código Penal (Ley 599 de 2000) en su artículo 122 el cual fue declarado exequible en la Sentencia C-355 de 2006, "en el entendido que no se incurre en delito de aborto cuando con la voluntad de la mujer, la interrupción del embarazo se produzca en los siguientes casos: i) Cuando la continuación del embarazo constituya peligro para la vida o la salud de la mujer, certificada por un medico; ii) Cuando exista grave malformación del feto que haga inviable su vida, certificada por un médico; y, iii) Cuando el embarazo sea el resultado de una conducta, debidamente denunciada, constitutiva de acceso carnal o acto sexual sin consentimiento, abusivo o de inseminación artificial o transferencia de ovulo 
fecundado no consentidas, o de incesto".

No obstante de entender la verdadera connotación sobre el tema de acuerdo a lo explicado en los acápites anteriores, para enfocar el estudio a la dinámica con la cual se ha venido desarrollando dentro de la opinión pública y los diferentes grupos que en él intervienen, se entenderá para todos los efectos, cuando se habla de legalización estamos refiriéndonos a que sea viable la permisibilidad de esta conducta dentro del ordenamiento jurídico y social colombiano, es decir, no este penalizada.

\section{UNA MIRADA RÁPIDA EN EL TIEMPO}

"El aborto es un problema secular en la historia de la humanidad. Existen datos que reportan que las interrupciones del embarazo se han producido en diversas épocas y, probablemente, en todos los lugares." (Bermudez Valdivia, 1998)

En Occidente hay algunos países que permiten el aborto, otros no y otros países que condicionan las circunstancias bajo las cuales se puede permitir, despenalizando en algunos casos, total o parcialmente tal conducta "delictiva". 
"El aborto es una figura de plena diversidad en su régimen normativo, pues marca un total contraste con lo que puede denominarse delitos clásicos, como son el homicidio, el asesinato y las lesiones en los delitos contra las personas. Todos ellos tienen una contemplación y un tratamiento homogéneo y cercano en casi todos los regímenes positivos; en contraste, el aborto voluntario es encajado de forma absolutamente dispar y heterogénea en todos los Estados del mundo, con un abanico de contrastes que no tiene paralelo en ninguna otra conducta humana". (Bermúdez Valdivia, La regulación jurídica del aborto en América Latina y el Caribe., 1998)

En este tema los antecedentes de estudio no son novedosos; realmente en Colombia siempre ha existido interés por el mismo e incluso han llegado a presentarse, desde el punto de vista legislativo, propuestas serias que plantearon posibilidades de regulación más coherentes con los hechos sociales y la realidad de cada época; En nuestro país, sin mencionar los esfuerzos en la comunidad internacional, se ha venido trabajando la idea de hacer algo en torno a él, pues las consecuencias de no tener una política justa, clara, adecuada y vanguardista para tratarlo, generan consecuencias realmente devastadoras en la sociedad colombiana.

Habiendo esclarecido los términos con los cuales podemos hacer referencia al 
tema del aborto en Colombia, es pertinente realizar un rápido recorrido en el que se puedan distinguir algunos referentes legislativos y judiciales en este tema, iniciando por indicar que desde el año de 1936 al aborto es contemplado en Colombia como un delito contra la vida y la integridad personal; desde entonces y tratando de hacer esfuerzos para solventar las consecuencias de este problema en la sociedad, se han argumentado en cada época y atendiendo en ocasiones a convencimientos propios y, en otras, a intereses partidistas, reformas legislativas y pronunciamientos judiciales que han marcado ciertos parámetros para llegar al punto jurídico en el que estamos hoy.

“Es así como el primer intento de discusión parlamentaria se efectuó en el año 1975 con el cual se buscaba reglamentar la interrupción terapéutica del embarazo por la demostrada ineficacia de la penalización" (Viveros, 1999). Posteriormente, en 1979, también se presentó una discusión con mayor trascendencia pues el proyecto que en este año se gestara, buscaba precisamente permitir la interrupción voluntaria del embarazo en cualquiera de estos tres casos: "cuando fuera consecuencia de acceso carnal violento o abusivo, cuando constituyera grave y actual o inminente peligro para la vida física o mental de la madre, que no fuera posible evitar de otra manera; cuando se comprobaran graves procesos patológicos o de malformación del feto" (Viveros, 1999). Era una medida tomada precisamente para evitar poner a las 
mujeres en condiciones de inseguridad y riesgo frente a la práctica de un aborto clandestino.

Entre 1989 y 1990, después de casi diez años de silencio frente al tema, hubo un nuevo intento de acción parlamentaria, en el que se buscaba la "legalización del aborto". En 1991, con ocasión de la Asamblea Nacional Constituyente y la consecuente Constitución Política de ese mismo año, se establece una protección a los derechos de la mujer, libre desarrollo de la personalidad, protección a la familia, a los jóvenes, al niño, al adolescente, y las personas de la tercera edad, con lo que queda establecido un nuevo panorama para abordar el tema del aborto.

En 1994 se sostuvo la prohibición del aborto, pero gracias a la coyuntura de las cumbres internacionales que se realizaron en ese año, se pudo determinar un nuevo matiz para el debate, lograr la participación de la academia en él y uno de los aspectos más importantes como era el designar el aborto inducido y clandestino como un problema prioritario de salud publica en América Latina, razones que serian claves para buscar un nuevo escenario de discusión política.

En 2006, se profirió la sentencia C-355 con la cual se declara exequible el artículo 122 de la Ley 599 de 2000, en el entendido que no se incurre en delito de aborto, cuando con la voluntad de la mujer, la interrupción del embarazo se produzca en 
los siguientes casos: I) Cuando la continuación del embarazo constituya peligro para la vida o la salud de la mujer, certificada por un médico; II) Cuando exista grave malformación del feto que haga inviable su vida, certificada por un médico;

y, III) Cuando el embarazo sea el resultado de una conducta, debidamente denunciada, constitutiva de acceso carnal o acto sexual sin consentimiento, abusivo o de inseminación artificial o transferencia de ovulo fecundado no consentidas, o de incesto.

En la actualidad sigue con vigencia la norma que penaliza el aborto como un delito contra la vida y la integridad personal, y por disposición de la Corte Constitucional, en sentencia de Constitucionalidad, se ha despenalizado la conducta en los tres casos mencionados anteriormente.

\section{EL DEBATE SOBRE EL ABORTO.}

Definir el aborto no es un asunto fácil; el concepto más básico que podemos formular, es aquel en el que se pone acento en la muerte deliberada de un embrión humano en desarrollo. Alrededor de esta afirmación se tejen versiones encontradas tanto para los que creen que tal interrupción debe estar permitida a las mujeres y por tanto consagrarse en un derecho protegido por el Estado (Proabortistas), como para aquellos que creen que dicha muerte es, en cualquier caso 
y bajo cualquier circunstancia, un asesinato (Pro-vidas). Caben, por supuesto, posturas intermedias, matices e incluso, posturas radicales.

Hay quienes en las posturas intermedias defienden la idea de que se debe permitir el aborto en ciertas circunstancias o bajo específicas condiciones, en las restantes, si se llegase a producir el aborto, hay que reprochar dicha conducta, moral y penalmente. Las posturas radicales son, también, en cierto sentido, interesantes: En el extremo de los argumentos pro-vidas encontramos recientemente la afirmación de que no sólo es un crimen reprochable, sino un "crimen de lesa humanidad", pues con el aborto se asesina a criaturas humanas indefensas. En el otro extremo, algunos pro-abortistas afirman que no sólo se debe permitir el aborto en circunstancias especiales, sino que se debe permitir en todos los casos en que la mujer embarazada lo decida, no importando el tiempo de la concepción o si los motivos que fundamentan la decisión son triviales, como un descuido deliberado en los métodos anticonceptivos, cambiar de pareja o no perder la figura.

\footnotetext{
${ }^{1}$ Nota de prensa. Periódico el Tiempo. $1^{\circ}$ de Octubre de 2010. "Desde Luego que materialmente lo es, formalmente las normas no lo consideran así, pero sociológicamente, ética y moralmente atentar contra la familia es atentar contra la humanidad" aseveró el Procurador Alejandro Ordoñez en la Instalación de Foro sobre la protección a la niñez. Con esta declaración se pone de manifiesto una versión radical pro vida. Lo curioso es que en tratándose de la disputa de derechos entre grupos sociales el Procurador General haya tomado una postura de tal naturaleza, con lo que el derecho consagrado para las mujeres en la Sentencia c355 de 2006 queda bajo la lupa moral (sino censura moral) de quien está llamado a protegerlo
} 
No se trata de deliberar entre estas posturas para determinar cuál es más acertada, como tampoco para tratar de conciliarlas, es bastante notoria la inconmensurabilidad moral, política y jurídica de los argumentos esgrimidos por cada grupo. El propósito, en cambio, es indagar por la eficacia de la normatividad sea cual sea el contenido que ésta tome. En esto resulta muy interesante el caso colombiano, pues en la normatividad vigente en nuestro país existen ambas formulaciones: Está vigente tanto la prohibición como la permisibilidad en casos específicos.

Dada esta circunstancia los ánimos están alterados por que como nunca hemos estado expuestos a tantos argumentos sobre el aborto, que van desde los moderados hasta los radicales. Aunque no es el centro de atención de este trabajo, haré una breve reflexión sobre la naturaleza de la discusión y por qué el nivel de radicalidad al que pueden llegar. Un texto en particular resulta de gran utilidad y pertinencia para el debate actual: "El dominio de la vida" del profesor Ronald Dworkin. Allí, el ilustre filósofo del derecho plantea una interesante cuestión: ¿Y si pro-abortistas y pro-vidas, pese a sus argumentos irreconciliables en realidad presuponen lo mismo?, y lo más importante ¿son tales presupuestos los que conducen a la radicalidad de los defensores de una u otra postura, no será pertinente replantear el punto de partida del debate? Dworkin explica porque no resultan creíbles los argumentos de quienes piensan que es posible un 
acuerdo entre abortistas y antiabortistas. Por lo general estos parten de la idea de que tales acuerdos deben respetar los principios fundamentales de la justicia, pero tales principios se ubican en posturas irreconciliables a los sujetos en discordia:

"Quienes creen, por ejemplo, que las mujeres tienen el derecho fundamental a tomar sus propias decisiones en materia de aborto, insisten en que cualquier solución aceptable debe respetar ese principio. Pero ninguna propuesta que respete tal principio podría ser aceptada en absoluto por las personas que creyeran que el aborto es un asesinato que viola los derechos e intereses más fundamentales de los niños no nacidos" (Dworkin, 1994, p. 17)

Él encuentra la dificultad de tales acuerdos en la pregunta sobre la cual gira el debate: “¿Es el feto un indefenso niño no nacido, con derechos e intereses propios desde el momento de la concepción?" Esta pregunta es altamente polarizadora y su respuesta, en cualquiera de sus sentidos, lleva a los sujetos a posiciones irreconciliables:

Si la respuesta es afirmativa, permitir el aborto es permitir el asesinato, $y$ abortar es peor que abandonar un niño molesto y dejarlo morir. Si es negativa, las personas que alegan ser "próvidas", o están actuando sobre 
la base de un profundo error, o son unos fanáticos puritanos y unos sádicos deseosos no de salvar vidas, sino de castigar a las mujeres por lo que ellas consideran un pecado sexual. (Dworkin, 1994, p. 17)

En la medida en que la discusión se plantea en esos términos, dice Dworkin, las dos partes no pueden razonar conjuntamente, pues no tienen sobre qué razonar o ser razonables. Ninguna de las partes ofrece un argumento favorable para la otra. Más, nadie habla con asesino de niños o se pone de acuerdo con ellos, así como tampoco, uno está dispuesto a llegar a acuerdos con "puritanos" cuyo propósito es castigar a las mujeres por su libertad sexual en lugar de salvar vidas.

Para Dworkin, esa manera convencional y pesimista de entender la naturaleza de la discusión es errónea y se basa en una confusión intelectual. Tal confusión conduce a tesis irreconciliables e impide acuerdos fundados en el respeto mutuo. Para aclarar este punto él clasifica las objeciones al aborto en dos tipos: "de carácter derivado" y "de carácter autónomo".

La objeción de carácter derivado presupone y deriva de la condición del feto, los derechos e intereses; y de tales derechos e intereses hace depender a su vez la intervención estatal: 
... abortar es inmoral porque viola el derecho de alguien a que no le maten, del mismo modo que matar a un adulto es normalmente inmoral porque viola su derecho a la vida. (Dworkin, 1994, p. 19)

La objeción de carácter autónomo parte del supuesto de que la vida tiene un valor intrínseco y por tanto, la razón para oponerse al aborto no se hace depender o derivar de la condición del feto, así como de "ningún interés o derecho particular":

“... la vida tiene un valor intrínseco, innato; que la vida humana es sagrada en sí misma, y que el carácter de sagrado de la vida humana empieza cuando se inicia la vida biológica, incluso antes de que la criatura -de cuya vida se trata- tenga movimiento, sensación, intereses o derechos propios". (Dworkin, 1994, p. 19)

Dworkin está pensando aquí en un argumento de oposición al aborto que no implique presuponer que el feto tiene intereses o derecho propios o que incluso se trata de una persona no nacida, pues, "... alguien que no considere al feto como una persona con derechos e intereses puede de esta manera oponerse al aborto exactamente con la misma energía que alguien que insista en lo contrario." (Dworkin, 1994, p. 21). La idea central de Dworkin es que casi todos los que se oponen al aborto lo hacen, en el fondo, por una razón de carácter autónomo y no por una de carácter derivado (Dworkin, 1994, p. 22). 
Aunque es posible creer que la objeción de carácter autónomo conduciría siempre a un rechazo total y radical del aborto, Dworkin cree que tal afirmación es incorrecta:

Es perfectamente coherente sostener esta idea [La objeción de carácter autónomo], incluso hasta el límite, y sin embargo creer que la decisión de poner fin o no a una vida humana durante la primera etapa del embarazo debería dejarse a la mujer embarazada, cuya conciencia está más directamente conectada con la decisión y que es titular de los intereses en juego más importantes.

Tal combinación de ideas es no sólo consistente en sí misma. Sino también congruente con la gran tradición de libertad de conciencia de las modernas democracias pluralistas. Es muy popular creer que no es de la incumbencia del Gobierno dictar lo que sus ciudadanos deben pensar acerca de valores éticos y espirituales, y especialmente, de valores religiosos. (Dworkin, 1994, p. 24)

La principal ventaja de centrar la atención entorno a la objeción de carácter autónomo, es según Dworkin, que permite que la oposición sea más consecuente consigo misma, al vincularla a “... una importante tradición de tolerancia religiosa que tiene fuertes raíces en todas las democracias modernas auténticas." y 
en especial, “... evita atribuir a la gente la idea, difícilmente comprensible, de que un organismo que no ha tenido nunca una vida psíquica puede sin embargo tener intereses" (Dworkin, 1994, p. 30). Para ilustrar la importancia de abandonar la objeción de carácter derivado y resaltar los argumentos implícitos (de carácter autónomo) Dworkin expone el siguiente ejemplo:

En la campaña Presidencial de 1992. Por ejemplo. Tanto el presidente Bush como el vicepresidente Quayle, quienes han expresado opiniones «pro vida» en los términos ortodoxos más duros, dijeron que apoyarían a su propia hija o nieta si decidiera abortar. Difícilmente harían tal cosa si de verdad creyeran que el aborto significara el asesinato de sus nietos o bisnietos. (Dworkin, 1994, p. 31)

Para Dworkin, las preguntas que deben estar en el centro del debate son distintas a la pregunta ambigua desde cuándo un feto es un ser humano. Estas preguntas son: ¿Cuándo adquiere intereses y derechos una criatura humana?, ¿Cuándo empieza la vida de una criatura humana a encarnar valor intrínseco y con qué consecuencias? Estas preguntas no exigen que se responda previamente la cuestión de sí el feto es un ser humano desde la concepción o si deviene como tal posteriormente en forma gradual o abruptamente. La pregunta de si el feto es una persona, es aún más engañosa que la pregunta por la condición humana del 
mismo ${ }^{2}$. Aunque, el uso de dicha expresión en el debate no es irrelevante. Quienes usan tal vocabulario "persona" quieren implicar, más explícita que implícitamente que, dado el concepto constitucional y civil de persona, el feto debe recibir el mismo tratamiento que reciben todas las personas, su muerte es por tanto un asesinato como lo sería la interrupción deliberada de la vida de cualquier persona, indistintamente si se trata de un niño o un adulto.

Es así como entonces, el debate sobre el aborto se muestra como una contraposición de argumentos irreconciliables. Las referencias a Ronald Dworkin han servido para mostrar la complejidad del mismo. Aunque como argumenta este autor, al parecer, el tema central no se encuentra en la condición del feto, en tanto si es o no un ser humano o persona, y en consecuencia de los intereses o derechos derivados de tal condición. Estas preguntas suelen ser engañosas y conducen a la radicalidad de las posiciones en el debate. Valdría centrar la atención en otro punto: “... cómo y por qué la vida humana tiene un valor

\footnotetext{
${ }^{2}$ Dworkin abandona la pregunta de si el feto es persona no porque ella sea incontestable o metafísica, sino porque es demasiado ambigua como para ser útil. El argumento que utiliza para mostrar la inutilidad de la pregunta es un poco chistoso: "Imaginemos que se descubre que los cerdos son mucho más inteligentes y emocionalmente complejos de lo que los zoólogos creen actualmente, y entonces alguien pregunta si. En consecuencia, un cerdo debería ser considerado una persona. [...] Podemos creer filosóficamente que los cerdos son personas. Pero que los seres humanos no tenemos ninguna razón para tratarlos del mismo modo como nos tratamos unos a otros; o, por el contrario, podríamos decidir que los cerdos no son personas desde el punto de vista de nuestra mejor interpretación de ese complejo concepto, pero que, no obstante sus capacidades les dan derecho a recibir el mismo trato que las personas se dan entre sí" (Dworkin, 1994, p. 34). Definir si el feto es o no persona no resuelve, ipso facto, el problema de cuál es el tratamiento que deba dársele, en cambio elude, según Dworkin, las preguntas claves que no dependen de tal clarificación.
} 
intrínseco, y sobre qué consecuencias se derivan de ello para las decisiones políticas y personales en materia de aborto." (Dworkin, 1994, p. 37)

A pesar de que estás preguntas son en sí mismas interesantes (tanto las preguntas tradicionales como las formuladas por Dworkin), el interés del tema, es otro: Una vez positivizada una determinada solución, como en el caso colombiano, las dos vías de regulación prohibición/permisibilidad, cuáles son los obstáculos que encuentran estas regulaciones y en qué medida afectan directamente la eficacia normativa. Además, qué relación tiene tal situación con el escenario decisional, entiéndase el órgano por el cual se ha producido la regulación normativa. La radicalidad de los debates y los obstáculos que encuentra dicha normatividad pueden estar relacionados con el tipo de regulación y la naturaleza del órgano que le dio origen ${ }^{3}$.

\footnotetext{
${ }^{3}$ La idea de relacionar eficacia de la normatividad con el tipo de órgano que la produce surge de la explicación del problema en Estados Unidos tal y como lo explica Ronald Dworkin, y en la cual se encuentra una curiosa similitud con el caso colombiano: "Muchos comentaristas insisten, sin embargo, en que la principal causa para que el debate del aborto haya provocado tanto enfrentamiento en Estados Unidos reside en la manera en que se formó el derecho estadounidense en materia del aborto. En otros países, la legislación sobre el aborto se creó mediante una variedad de pactos legislativos y políticos. En Estados Unidos, en cambio, la legislación no se impuso tras una lucha y acuerdo políticos, sino por el simple imperativo de la Corte Suprema (Tribunal Supremo). De acuerdo con la Constitución, la Corte tiene el poder de declarar inconstitucionales las leyes adoptadas por el Congreso federal o por cualquiera de los estados, esto es, que pueden invalidarse por contradecir las limitaciones que la Constitución impone a los poderes públicos. Una vez que la Corte Suprema ha fallado, ningún otro poder del Estado puede contravenir su decisión, sin importar cuán grande sea la mayoría popular que se oponga a la misma. Ciertamente, el pueblo puede revocar una decisión de la Corte Suprema por la vía de reformar la Constitución y así conferir explícitamente a las asambleas legislativas el poder del que-según la Corte Suprema-carecen. Pero esto es algo extraordinariamente difícil, y en la práctica, los políticos que aborrecen una determinada sentencia de la Corte Suprema sólo pueden esperar que se nombren nuevos jueces que estén de acuerdo con ellos, y que
} 
Dicho ejercicio implica comprender los tipos de regulación sobre el aborto y los órganos que han intervenido en dicha producción normativa.

\section{RUTAS NORMATIVAS SOBRE EL ABORTO EN COLOMBIA Y EL PROBLEMA DE LA EFICACIA.}

Abordar la eficacia de la normatividad sobre el aborto en Colombia presupone tener bien claro dos rutas normativas: Por un lado, la regla general que hace tradición, en tanto es la línea sostenida en la legislación penal, con algunos matices en cuanto a la pena y a las circunstancia que concurren a la conducta punible. Por otro lado, la excepción (o excepciones) establecidas por la sentencia C-355 de 2006 y que en alguna medida eclipsa, en los debates sobre el tema, a la regla general. La existencia de dos rutas jurídicas opuestas (prohibiciónpenalización/permisibilidad-despenalización) implica dos tipos de perspectivas acerca de la eficacia jurídica.

En la primera, el problema de eficacia ha estado centrado en unas normas que penalizan la práctica del aborto, y sólo por vía de atenuantes punitivos, se toman en cuenta las específicas circunstancias personales o contextuales de cada

un día la Corte Suprema así renovada revoque su propio precedente judicial, algo que está autorizada a hacer." (Dworkin, 1994, p. 13). 
caso. El problema de eficacia, así, está centrado en la práctica clandestina de los abortos y en la incapacidad del Estado, en términos generales, para identificar y procesar a los delincuentes. A pesar de la existencia de un notorio rechazo social (en su mayor parte de tipo religioso) la pregunta clave sigue siendo, qué factores normativos o sociales dificultan la efectividad de la prohibición.

En la segunda, el problema de eficacia ya no está relacionado con sancionar a quienes practiquen abortos, sino por el contrario, hacer efectivo la práctica del mismo en los casos autorizados por el mandato jurídico de la sentencia C-355 de 2006, e incluso, sancionar (sin que aún esté muy claro la naturaleza de la sanción) a quienes impidan, dificulten o pongan obstáculos para la práctica de la misma. EFICACIA Y REGLA GENERAL DE PENALIZACIÓN DEL ABORTO.

Tal como están dadas las condiciones actuales en el ordenamiento jurídico colombiano, una mujer que permita que se le practique un aborto y/o quien intervenga en tal hecho, es susceptible de ser investigado y juzgado penalmente por parte del Estado, pues incurre en la comisión de esta conducta legalmente delictiva, de lo que se desprende que la eficacia de la norma penal es eminentemente policiva pues, el Estado deberá, a través de los órganos competentes y los procedimientos establecidos legítimamente, buscar en todos los casos la imposición de una sanción al delincuente, tal como sucede en todos 
los casos donde se tipifique una conducta prohibida por la legislación.

La regulación sancionatoria del Estado establecida en la Ley 599 de 2000, determina su límite para perseguir procesalmente a las mujeres que abortan bajo cualquier circunstancia no permitida por la ley, y con la imposición de una pena, garantiza la protección de los bienes jurídicos que se amparan con el tipo penal del aborto. Esta posibilidad, es decir, la de actuar con funciones policivas con la imposición de sanciones penales, "supone siempre una grave injerencia del Estado en los derechos fundamentales de los potenciales autores del delito" (Laurenzo Copello, 1990), sin perjuicio del desconocimiento también de otras circunstancias que determinan la complejidad de la eficacia en la práctica.

Para explicar la premisa citada, es menester entender que la función estatal sancionatoria limita los derechos fundamentales, sobre todo, las libertades individuales cuando prohíbe a la mujer determinarse para tomar decisiones respecto de si tener un hijo o no; pero al tiempo le impone un estigma como una delincuente, independientemente de las circunstancias que la motivan para realizar el hecho, y al tiempo, desatiende sus razones.

Esta función policiva a la que se circunscribe la norma penal se ve afectada por la incapacidad del Estado para lograr su eficacia en la práctica, toda vez que las 
condiciones de clandestinidad $^{4}$ en las que dichas conductas se llevan a cabo recurrentemente, aún incluso, después de la despenalización parcial del delito, no permiten que haya una correspondencia entre la norma prohibitiva y la sanción a los sujetos que cometen el hecho.

La situación de clandestinidad limita la posibilidad de conocer con certeza primero, la realidad del número de abortos que realizan en Colombia, los casos de reincidencia y si es una práctica habitual en algunas mujeres. La norma penal en este sentido no es efectiva ni eficaz, en tanto y en cuanto la violación a la prohibición que establece el artículo 122 de la ley 599 de 2000, es patente. El número de muertes reportadas por esta causa así como también por el número de

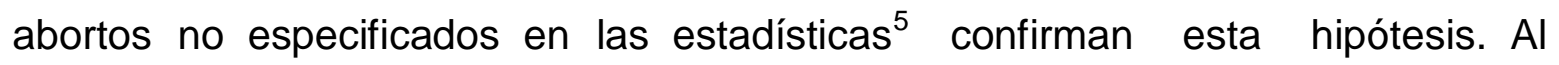
respecto afirma el profesor Marthe Zapata:

\footnotetext{
${ }^{4}$ Prada Salas, Elena. Aborto complicado: La punta del iceberg de la práctica clandestina. “....Finalmente es necesario considerar que mientras el aborto continúe siendo ilegal en Colombia, la verdadera incidencia y magnitud del aborto inducido no se conocerá y las estadísticas oficiales de los egresos hospitalarios solo mostrarán la punta del iceberg de la práctica clandestina. Y mientras continúen vigentes leyes fuertemente restrictivas, las desigualdades sociales tenderán a ahondarse, si se tiene en cuenta que las mujeres que no pueden acceder a servicios seguros son las que en mayor proporción enfrentan el riesgo de complicaciones, si no de muerte...."

${ }^{5}$ Prada Salas, Elena. Aborto complicado: La punta del iceberg de la práctica clandestina. “...En la practica la aceptación de un aborto inducido, no se da y no se puede dar, debido a la restrictiva legislación colombiana que contempla penas tanto para quien provoca un aborto como para quien permite o solicita, se le provoque. Exige al mismo tiempo, a quien atiende la complicación, la denuncia del caso ante la autoridad competente. Debido a estas circunstancias las mujeres casi nunca reconocen en el momento de la hospitalización haber sido sometidas a una maniobra abortiva, ni delatan a quien les ayudó en un momento de necesidad. Por su parte el equipo de salud, se abstiene en la mayoria de los casos de hacer la denuncia por falta de la evidencia clínica exacta, por los trámites burocráticos que ello demanda y quizás también por razones de solidaridad de agregar un problema más a la mujer que, ya de por sí, enfrenta serios quebrantos en su salud física, emocional, social y familiar..."
} 
"El aborto es un mal real, de dimensiones enormes, desconocidas en toda su magnitud, precisamente porque es clandestino. Sus causas no son solamente fallas personales a la moralidad individual. Está incrustado profundamente en la situación socio-económica de la población y es, en buena parte, consecuencia de males sociales más profundos. Por supuesto, el remedio del aborto y de los males que lo causan, no consiste en legalizarlo. Pero legalizarlo permite conocer su dimensión y sus causas, y permite remediar los males subyacentes que su clandestinidad produce, como enfermedades, muertes, infecciones, explotación de mujeres, irresponsabilidad masculina, abusos económicos, practica clandestina y criminal de una medicina sin higiene y sin control, existencia de clínicas ilegales, situaciones desesperadas, etc., etc. En una palabra, proliferación submarina de un crimen generalizado..."

Esta circunstancia sólo nos ayuda a comprender que, el Estado es incapaz de evitar o disminuir la concurrencia de la conducta delictiva del aborto. El efecto policivo de dicha normatividad no pone fin ni le pondrá, a una figura como la del aborto mientras subsistan situaciones que conlleven a la mujer a tomar este tipo de decisiones, sobre todo en un país como Colombia en el que confluyen toda una serie de condiciones, que ayudan a que el contexto de las mujeres sea 
conflictivo y difícil.

Tal como se ha manifestado, para hablar de eficacia, es necesario observar que grado de cumplimiento tiene la norma en los sujetos a los cuales está dirigida su aplicación; la sospecha es precisamente que los sujetos, en el caso del aborto, tienen razones que los llevan al incumplimiento forzado de la norma aunque sea prohibitiva y en consecuencia, sancionatoria, dado que dichas razones están íntimamente ligadas con situaciones que obligatoriamente conducen al sujeto al no acatamiento o incumplimiento de lo prescrito en el ordenamiento jurídico.

Es aquí donde confluyen la mayor parte de las circunstancias que dificultan la aplicación eficaz de la prohibición. Colombia es un país con problemas de pobreza extrema, de miseria inclusive; las mujeres, aún con toda la protección que brinda la Constitución política de 1991, son sometidas a discriminación constante de toda índole; se les reconoce escasamente el trabajo doméstico que realizan y en el que se encuentra un porcentaje muy amplio de la población femenina colombiana; las condiciones de pobreza, miseria e indigencia de las mujeres las lleva a la imposibilidad de planear, proyectar y construir un modelo de vida que les permita realizar sus más sencillos sueños, sin contar con que un gran número de ellas son madres responsables cabezas de hogar, con el agravante de la desproporción laboral a la que en ocasiones se ven sometidas respecto de los 
hombres y los altos índices de desempleo que no facilitan su labor.

Es decir, parecería que tales circunstancias confluyen en poner a la mujer en una situación excusable en la determinación que tiene a la hora de tomar la decisión de abortar; lo cierto es que, todas ellas atienden a lo que constituye la causa de su decisión y por ende, mientras tales condiciones no desaparezcan la mujer en estado de embarazo, que se vea avocada a una necesidad de tales magnitudes, acudirá a la posibilidad de abortar, restándole importancia, como lo ha hecho hasta ahora, a la vigencia de una norma penal que por esta razón, es ineficaz, pues las circunstancias traen como consecuencia que el acatamiento por parte de quienes están llamados al cumplimiento de la norma jurídica no se sometan a ella, independientemente de las consecuencias punitivas que aquellas tengan.

Al respecto se pronuncia en el siguiente sentido (Marthe Zapata, 1994):

“...Nuestro Código Penal en relación con la política del aborto ha sido impotente para contrarrestar o combatir el problema. Lo mismo ha ocurrido en la mayoría de los países del mundo; la amenaza penal es impotente como medio para disuadir o combatir el aborto; nunca ha tenido éxito ni lo tendrá, pues las circunstancias que obligan a la mujer a violar la ley son superiores a las amenazas de la justicia. Casi siempre el delito escapa a 
las sanciones penales porque es difícil en la gran mayoría de los casos, demostrar que se trata de un aborto inducido (criminal)."

Lo cierto es que es poco creíble que las mujeres en general estén de acuerdo con abortar e incluso no es la mujer de mala reputación la que acostumbra recurrir al aborto ni la que toma esta clase de decisiones, precisamente porque no es su estatus o condición lo que la determina que lo haga; una mujer no aborta en ningún país del mundo y menos en Colombia con la tradición católica que nos antecede, porque le guste abortar o por que disfrute hacerlo; una mujer toma la decisión de abortar porque hechos trascendentales en su vida la llevan a hacerlo; no es la moral la que se lo impide o la que la impulsa, por el contrario, es en este terreno justamente en donde las causas que ella tiene para terminar con su embarazo cobran validez.

Los médicos dan cuenta de lo desagradable y lamentable de la situación del aborto, para ellos es una acto que representa una contradicción con el objeto de defensa de su profesión, es decir, de la vida. Y la mujer, por más desacuerdo que tenga con este hecho delictivo termina tomando la decisión porque para ella se trata de circunstancias coyunturales que no puede ni debe afrontar. En este sentido, la moral no pesa sobre el aborto: una mujer que tiene una estructura moral y ética formada en valores, no deja de abortar por este hecho; lo 
hace aún en contra de ellos y a sabiendas incluso que infringe la ley, por un hecho más poderoso y relevante en ese momento de su vida, lo cual lo constituyen sus razones o causas; este hecho no la convierte en una mujer inmoral y mientras persistan éstas, el aborto subsistirá no obstante la penalización de la conducta haciendo a la norma permanentemente ineficaz por la falta de correspondencia, acatamiento y cumplimiento que exige el orden jurídico para que sus normas sean tenidas como tal; la situación se complica cuando como consecuencia de la prohibición, resulta la práctica abortiva en circunstancias de clandestinidad que agravan los hechos, pues la mujer es sometida a peligros poniendo en riesgo su vida, y de paso deja sin efectos la estructura garante de derechos fundamentales que ella ha logrado reivindicar a lo largo de la historia.

\section{EFICACIA, EXCEPCIONES Y DESPENALIZACIÓN PARCIAL.}

Al tiempo que existen hechos que no permiten efectivizar las normas de regulación general en la práctica, también existen hechos que polarizan la aplicabilidad de las normas que regulan la despenalización del aborto referidos a los casos particulares que la Corte Constitucional autorizó en la sentencia C-355 de 2006, y que a juicio de quienes están en contra de la decisión, consideran los tres casos como una fuga a la regla general de penalización. 
Los tres casos de despenalización autorizados por la Corte Constitucional constituyen una posibilidad de introducir a nuestra legislación en una corriente más permisiva, sin embargo, la Corte, en esta decisión deja claro que sólo son exclusivamente tres los casos en los que una mujer puede legítimamente abortar en nuestro país, lo cual nos indica que los demás casos que pueden generar en la mujer la decisión de abortar, por muy graves y determinantes que sean en su vida, no son válidos porque la Corte estimó como los más relevantes aquellos que se consagraron en dicha sentencia como las excepciones a la regla general de penalización. El hecho es que, no obstante estar permitido en los tres casos autorizados por la justicia colombiana, también en ellos se dificulta su aplicabilidad en la práctica, constituyendo este, un caso más grave que la ineficacia generada en la norma penal general, pues aún después de haberse realizado el reconocimiento de su derecho a decidir abortar en circunstancias especiales, encuentra una dificultad de grandes proporciones al momento de llevar a cabo la práctica abortiva autorizada. Este también constituye un problema de eficacia normativa que analizaremos a continuación.

Los obstáculos que constituyen un verdadero freno al momento de hacer efectiva la norma jurisprudencial, son de orden social y moral, y básicamente los debates en cuanto a esa inoperancia en la que se traduce la ineficacia de dicha norma, se realizan en el sistema de salud y en el sistema educativo. Es por esta 
razón que hoy, después de todo el recorrido avanzado en el camino de la despenalización parcial, todavía nos encontramos con realidades como la que ocasionó el pronunciamiento de la Corte Constitucional en sentencia T-388 de 2009, caso en el cual se refleja no sólo la resistencia al cumplimiento de la orden impartida por este alto tribunal, sino también la inadecuada o intencionada interpretación que los diferentes actores le han dado a los alcances de este histórico pronunciamiento.

El envió de una carta de la Procuraduría a la Superintendencia de Salud en donde se manifiesta que la C-355/06 despenalizo parcialmente el aborto pero no convirtió al aborto en un derecho, y por lo tanto, los funcionarios de la salud no tienen la obligación de remover obstáculos para dicha práctica, constituyen un discurso que resulta contrario a la orden de la Corte Constitucional y que pone de presente en forma manifiesta hechos que generan la ineficacia de la Jurisprudencia Constitucional.

Así mismo sucede con la orden que se impartió en la sentencia T-388 de 2009 respecto de iniciar campañas de información educativa que se establecieran en todos los colegios para difundir enseñanza sobre los derechos sexuales y reproductivos de la mujer; Consultando aleatoriamente varios colegios de la ciudad de Bogotá, nos encontramos que en ninguno de ellos se ha efectuado 
cambios en los pensum académicos, planes de estudios y/o sílabos de las asignaturas o cursos que allí se regentan, encaminados a proporcionar información detallada sobre el nuevo panorama normativo en Colombia respecto del aborto y los derechos de las mujeres, en razón a que, en algunos casos manifiestan no haber recibido orden, directriz, resolución o instrucción para llevar a cabo tal fin y en otros casos, por que se limitan a enseñar lo tradicional de la educación sexual de acuerdo a los principios de autonomía de los planteles estudiantiles. Hay instituciones que manifiestan que hasta la fecha no han hecho inclusión dentro de sus programas académicos ninguna información que se direccione a capacitar a los estudiantes sobre los derechos sexuales y reproductivos de la mujer, y en forma específica sobre el aborto; solo han centrado su educación respecto de estos derechos, en forma genérica, llegando a dar instrucciones sobre las especificaciones de la píldora del día después.

Se presenta entonces una situación ambivalente pero bastante curiosa en el caso del aborto actualmente; y es el referido a que, por un lado estamos en la lucha inagotable por evitar que las mujeres aborten y sancionar a las que realicen dicha conducta, pero por otro lado, estamos en la lucha interminable para lograr que las mujeres que quieran abortar y estén autorizadas por el ordenamiento jurídico para hacerlo, puedan hacerlo sin restricciones, impedimentos y señalamientos de índole alguna que obstaculicen esa posibilidad, como aquellas que se han citado. 
Cuando una mujer está inmersa en alguna de las circunstancias autorizadas por la ley y decide abortar bajo la estricta protección de su derecho fundamental por parte del Estado, encuentra un sin número de impedimentos que entorpecen la materialización de su derecho fundamental. Estos van desde el señalamiento y estigmatización a la que se ven sometidas en algunas instituciones de salud, que no obstante de llevar a cabo la práctica, realizan trámites discriminatorios haciendo más gravosa y sentida una situación que por más autorizada que esté por la norma, no deja de ser difícil, sobre todo en tratándose de mujeres con arraigos religiosos y axiológicos que le hacen más traumática su decisión, hasta también, la posición equivoca de funcionarios de la salud que, al igual que funcionarios judiciales como los que cita la sentencia T388 de 2009, hacen una interpretación particular de lo ordenado por la Corte para evadir el cumplimiento del fallo e impedir la materialización de un derecho legalmente reconocido en nuestro país ${ }^{6}$.

En este punto vale la pena detenernos un poco con el fin de identificar los principales obstáculos que impiden la eficacia de la orden de la Corte y observar

\footnotetext{
${ }^{6}$ Informe Sombra Colombia al Comité de la CEDAW. “....Sin embargo, no obstante que el fallo de la Corte es vinculante y de obligatorio cumplimiento desde el mismo día de su notificación se presentan innumerables inconvenientes para que las mujeres accedan efectivamente a la práctica del aborto en las tres circunstancias en que ha sido despenalizado, dado que en muchos casos las directivas, profesionales y trabajadores vinculados al sistema de salud se muestran renuentes a cumplir con sus deberes, aún en casos verdaderamente extremos, en los que se comprometen derechos de los menores que, como se sabe, son prevalentes."
} 
cuáles son las posibles razones de quienes protagonizan los obstáculos al cumplimiento de la norma, y si estas, están relacionadas con la vulneración de también derechos fundamentales, más exactamente en el caso de los médicos, jueces, la iglesia y entidades del Estado.

Más allá de si está permitido o no el aborto, importa considerar la trascendencia del escenario al cual se traslada la toma de decisiones. Debe decidirse en democracia, tal como si se tratara de un asunto de deliberación pública propia de una asamblea o debe dejarse la decisión última a la mujer, entendida esta como aislada de todo contexto social, o de la mujer en compañía de su pareja sexual, de sus padres, de un psicólogo, del ministro de la iglesia, etc.

En el caso colombiano se puede observar con claridad esta doble perspectiva: La ley penal parece escenificar la toma de decisiones propia de una sociedad democrática que hace penetrar los argumentos morales de la mayoría en las disposiciones normativas que han de regular la conducta de los sujetos. No hay nada de extraño en ello, además, tal enfoque explicaría la constante apelación de los opositores a la sentencia C-355 de 2006 y del aborto en general, al argumento de mayorías religiosas católicas o cristianas. En tal sentido, el escenario propicio para definir la permisión o prohibición es el más parecido a la "asamblea deliberativa de las mayorías". En cambio, la sentencia de la Corte escenifica la 
toma de decisiones sobre el aborto en un espacio más reducido o restringido; "la soledad de la mujer". Es a ella y sólo a ella, en determinados casos, a la que le corresponde tomar la decisión teniendo en cuenta su conciencia o sus intereses.

La dualidad de enfoques coincide con la dualidad normativa, la primera con la vía legislativa y la segunda con una regulación que no surge de un procedimiento democrático, en el sentido de la deliberación plebiscitaria (contra mayoritaria). Esto puede explicar los problemas de resistencia actual de muchos grupos opositores a la decisión: Un órgano que atiende razones puramente legales y doctrinales de raíces liberales, la Corte Constitucional, ha establecido una visión sobre el aborto "ajena a las mayorías nacionales", y con ello ha cambiado o trocado, para casos específicos, el escenario último en el que se toman las decisiones. Además explica el cambio en los ámbitos del debate, de las cámaras legislativas (legislación) a las clínicas y establecimientos educativos.

En las apreciaciones anteriores se encuentra la principal justificación de los temas abordados en este trabajo, el cual busca no solo identificar los escenarios donde se realizan los debates sobre el aborto y los factores que impiden su eficacia en la práctica, sino que con estos dos focos de estudio, se establece la dependencia y correlatividad de lo primero y lo segundo, en el sentido de que para pretender un grado de eficacia del aborto en Colombia mínimamente aceptable, es necesario 
que los escenarios en donde se lleven a cabo tanto el debate como aquel donde se toman las decisiones en cuanto al problema, sean participativos 0 mayoritarios.

No obstante de reconocer la legitimidad y trascendencia de la decisión de la Corte Constitucional, se plantean en lo sucesivo, ciertas inquietudes que surgen como consecuencia del análisis que sobre los escenarios de decisión se establecen en este trabajo en cuanto al aborto, con el único ánimo de propiciar nuevos puntos de análisis e investigación posterior. En razón a esto, un asunto de gran importancia escapa a la visión solipsista de los derechos fundamentales de la mujer.

Como puede observarse el derecho de objeción de conciencia es un correlato del derecho de libertad de conciencia, también constitucionalizado y protegido por nuestro ordenamiento jurídico. De allí que el reconocimiento del derecho a las mujeres para que estas puedan decidir, en los casos excepcionales contemplados por la sentencia de marras, interrumpir la concepción, haya chocado con este otro que alegan tener profesionales del sector salud, e incluso, ahora profesionales del sector educativo a propósito de la formación sexual en el sistema educativo.

No es desconocido por nadie que el sector social que promueve en sentido fuerte 
este tipo de reivindicaciones es la Iglesia Católica. La Iglesia Católica posee una larga tradición doctrinal en materia de objeción de conciencia, esto debido a las enseñanzas del cristianismo sobre los dos poderes: el divino y el secular. Para la Iglesia la conciencia es ese espacio santo donde Dios habla con el hombre y en el que ningún poder humano, entiéndase civil o social, puede o debe interferir.

A diferencia de la libertad de conciencia civil de la que hemos hablado anteriormente, la libertad de decisión en la Iglesia Católica entraña una paradoja, el hombre tiene dignidad en la medida que su libre conciencia no es absolutamente libre:

"La Iglesia considera sagradas la dignidad de la conciencia y "su libre decisión" (GS 41b), mas no porque esta decisión sea autónoma, sino al contrario, porque alli, "en lo profundo de la conciencia el hombre descubre una ley que él no se da a sí mismo pero que debe obedecer, y cuya voz lo llama siempre a amar y hacer el bien y a huir del mal" (La Iglesia Católica y la Objeción de Conciencia. Martin de Agar, en: Pacheco Escobedo, 1998, p. 239)

Desde esta visión de la libertad de la conciencia, las leyes que permiten el aborto y otras conductas como la eutanasia, el matrimonio homosexual y el divorcio no son progresistas ni libertarias: 
La sociedad plural en que vivimos se orienta con frecuencia en sentido contrario a la moral cristiana; por su parte, las llamadas leyes permisivas tantas veces no se muestran tales en la práctica, sino que, de hecho, imponen (a pesar de la tolerancia en que dicen inspirarse) acciones que van contra la conciencia. Los católicos tienen el deber de respetar la conciencia de los demás, pero al mismo tiempo tienen el deber de servirse de los medios legítimos a su alcance para salvar su conciencia. (La Iglesia Católica y la Objeción de Conciencia. Martin de Agar, en: Pacheco Escobedo, 1998, p. 244)

El asunto para efectos de la eficacia este tipo de objeciones no tendrían que amenazar con volverse un obstáculo serio a la eficacia de la despenalización parcial a no ser por la extensión del argumento de objeción del plano individual al colectivo, incluyendo éste establecimientos de salud y educativos en manos de la Iglesia Católica y otras confesionalidades:

"La objeción de conciencia es básicamente personal como lo es la conciencia en nombre de la cual se ejerce. De todas formas, así como la libertad de conciencia puede presentar aspectos colectivos e institucionales, sobre todo cuando está conectada con una determinada 
confesión, también una suerte de objeción puede ser ejercida por las mismas confesiones y por otras entidades de inspiración religiosa, sea para salvaguardar su propia identidad, sea para garantizar la conciencia de Iso que en ellas trabajan $y$ de quienes recurren a sus servicios. Estas instituciones, por el deber de coherencia que sobre ellas incumbe, tienen el derecho a negar su colaboración en actividades impuestas por las leyes que sean contrarias a su ideario". (La Iglesia Católica y la Objeción de Conciencia. Martin de Agar, en: Pacheco Escobedo, 1998, p. 245)

Extender el derecho de objeción de conciencia no sólo a los sujetos, para los cuales es un derecho irrenunciable, salvo los límites a todo derecho en el seno de un Estado de derecho de raíces liberales, constituye una autentica amenaza debido al control que las confesionalidades, en particular las de orientación católica tienen sobre establecimientos de salud y centros educativos.

No deja de producir perplejidad que la Iglesia Católica se oponga tan virulentamente al aborto, pero haga relativo silencio frente a otras formas de afectación de la vida tales como homicidios, masacres, participación de sus feligreses en grupos armados (incluido el Estatal). Para ello los autores católicos han distinguido entre objeciones de conciencia obligatorias ${ }^{7}$ y facultativas $^{8}$. Al

\footnotetext{
7 "Es obligatoria aquella objeción que viene exigida para todos por doctrina moral cristiana, en cuanto corresponde a un precepto básico y unívoco de la ley divina, como el "no matarás", tal es, por ejemplo, la
} 
margen de esta posible inconsecuencia de las confesionalides en general y no sólo de la Iglesia Católica, preocupa que una extensión del derecho a la libertad de conciencia y su correlato objeción, se conviertan en la forma de obstaculizar el derecho de las mujeres, incluso de las que no son religiosas, por parte de quienes han sido autorizados por el Estado, bajo sus leyes y supervisión a prestar un servicio público esencial.

\section{CONCLUSIONES}

Abortar o practicar un aborto, es una conducta que ha estado penalmente prohibida en nuestro ordenamiento jurídico desde los orígenes de nuestro sistema penal. Hoy, bajo un marco constitucional amplio y vanguardista, la Corte Constitucional ha establecido una particular regulación dual, pues por un lado se sostiene la prohibición y se penaliza la conducta del aborto y de otra parte, se despenaliza la misma, permitiendo su causación en tres casos puntuales: cuando el embarazo constituya peligro para la vida y salud de la madre, la inviabilidad extrauterina del feto y cuando el embarazo haya sido producto de violación o incesto.

objeción al aborto o cualquier práctica contra la vida de un inocente" (La Iglesia Católica y la Objeción de Conciencia. Martin de Agar, en: Pacheco Escobedo, 1998, p. 242)

8 "Sería facultativa la objeción de conciencia al servicio militar, porque el bien de la paz y el rechazo en principio de la violencia no prohíben el recurso a la misma cuando no haya más remedio". (La Iglesia Católica y la Objeción de Conciencia. Martin de Agar, en: Pacheco Escobedo, 1998, p. 243) 
El problema, como hemos visto, radica en el juicio de eficacia que difiere para las dos vías de regulación. Creo que la normatividad sobre el aborto resulta ineficaz en algunos casos, que resulta dispendiosa su aplicación en la práctica, dado su doble finalidad de prohibir o permitir una conducta que fenomenológicamente son similares y que en particular, le corresponde a las autoridades asumir.

En el caso de la prohibición, continúan realizándose abortos clandestinos con todas las consecuencias que este hecho conlleva y la acción policiva del Estado al penalizar queda reducida a prohibir y penalizar y, en el segundo caso, que es el más interesante desde el punto de vista jurídico, existe una fuerte resistencia a cumplir con el mandato constitucional por parte de funcionarios judiciales y de los profesionales de la salud, que perteneciendo a la red hospitalaria, y que estando obligados, como todos los particulares al cumplimiento de las normas, evaden el cumplimiento de este mandato, impulsando a las mujeres en algunas ocasiones a someterse a abortos clandestinos o en otras, a tener que avanzar en un embarazo que por autorización judicial están legitimadas para interrumpir.

Tales hechos son constantes y no logra percibirse que puedan dejar de ocurrir en la medida en que hay inconformidad en el tratamiento de derechos que con las decisiones de la Corte, emanadas a partir de la sentencia c-355 de 2006, 
específicamente la Sentencia T-388 de 2009, han sido socavados, generando en quienes están afectados con tales decisiones, una renuencia al reconocimiento de los derechos de la mujer y en consecuencia la oposición abierta a la práctica de la $\operatorname{IVE}^{9}$, evidenciando ausencia de acatamiento y cumplimiento de las normas, lo que se traduce, tal como se explico, en ineficacia normativa. Es claro que la dificultad para asumir las decisiones sobre este problema no son fáciles; la metáfora de las "arenas movedizas" que se ha utilizado en acápites iniciales de este trabajo, es ventajosa para hacer referencia a esta situación, en la que por donde tratemos de dilucidar es factible que resolvamos ventajosamente unos derechos, poniendo en una situación contrariamente perjudicial a otros.

Después de la Sentencia T-388 de 2009 el debate sobre el aborto se trasladó a dos sectores: salud y educación; encontrando en el primero serios obstáculos que poco a poco desdibujan la posibilidad de eficacia de la orden constitucional, en la medida en que es allí donde tiene su accionar la práctica de la IVE y hay una pública renuencia a realizar tales procedimientos, por cuanto con las decisiones de la Corte se ha impuesto una obligación de respeto ante derechos sexuales y reproductivos de la mujer, cuya materialización en la práctica supone una vulneración concomitante al derecho de libertad de conciencia de los médicos, que de acuerdo a sus creencias valorativas y convicciones, no

\footnotetext{
${ }^{9}$ Interrupción voluntaria del embarazo
} 
pueden ejecutar este tipo de procedimientos y si bien es cierto que tal figura no puede constituir una talanquera para la consecución de la interrupción del embarazo en los casos autorizados por la Corte, tampoco de ella se debe desconocer su desarrollo en el ejercicio médico ya que en la medida en que esto suceda, siempre habrá una negación a la práctica de los abortos, porque nadie cede ante sus convicciones y menos cuando media un tratamiento desproporcional en la protección de derechos fundamentales.

“...Se debe efectuar por parte de los operadores jurídicos, los juristas, la academia, las instituciones de salud y el personal médico un análisis hermenéutico legal, tendiente a una correcta aplicación de la sentencia, ya que la finalidad de la Corte no es permitir que la mujer aborte, sino salvaguardar los derechos fundamentales". (Pabón Giraldo, 2008, p. 82)

La Corte Constitucional ha impartido claras instrucciones a los organismos del Estado, en el sentido de difundir a través de campañas educativas todo lo necesario para empoderar en la juventud y en general, en la sociedad colombiana, todo la información que sea menester en relación con los derechos sexuales y reproductivos de la mujer, lo cual también ha encontrado dificultad para materializarse y lograr que dicha decisión goce de eficacia, toda vez que 
hay una franca oposición ${ }^{10}$ por parte de un sector de dichas entidades para cumplir con la orden constitucional, con argumentos de defensa del derecho a la vida, el cual esta salvaguardado por el ordenamiento jurídico, no solo de manera amplia en pronunciamientos anteriores y en la misma sentencia C-355 de 2006, sino también en fallos posteriores a ella.

Esta defensa a tal derecho no solo es predicable de este alto Tribunal, pues refiriéndonos al caso especifico del Ministerio Publico, ha sido la Procuraduría General de la Nación, quien con firmeza ha defendido el derecho a la vida no obstante de que en la administración del actual Procurador dicha protección obedece no solo a las funciones propias del cargo sino, tal como en repetidas ocasiones lo ha manifestado, a convicciones y creencias personales y religiosas. Similar situación se presenta también en los planteles educativos que se han declarado confesionales y que por razones de credo, religión, principios y convicciones propias en el tema del aborto, no transmiten la información a sus estudiantes en las condiciones en que ha ordenado la Corte. Estos hechos

\footnotetext{
${ }^{10}$ Nota de prensa del Periódico el Tiempo. $1^{\circ}$ de Diciembre de 2010. "En una decisión conocida por EL TIEMPO, la Corte Constitucional le hizo un fuerte llamado de atención al procurador Alejandro Ordóñez por "desacatar" órdenes dadas por ese mismo Tribunal sobre la implementación de una 'cátedra' sobre el derecho al aborto y los derechos sexuales de las mujeres. Según la Corte, la Procuraduría no ha cumplido con garantizar las campañas masivas en entidades educativas sobre ese tema. "Está incumpliendo funciones constitucionales", le advierten los magistrados al Procurador, abierto crítico del aborto y quien criticó desde el primer momento esa cátedra."Si el incumplimiento se debe a razones morales, (el Procurador) debería expresarlo fundadamente y apartarse, para que otro funcionario público lo haga", dice un ato de la Corte Constitucional. Además, señala que la omisión "puede derivar en la comisión de delitos o faltas disciplinarias".
} 
demuestran que a pesar, que el aborto es un problema que no se decide desde lo moral, tiene matices morales que polarizan el tratamiento jurídico del tema y su eficacia normativa.

Las normas que regulan el aborto en Colombia serán ineficaces (no en todos los casos) mientras existan factores externos a ella que determinen su no acatamiento en una gran parte de los asociados y, en consecuencia, incumplimiento, derivado éste por la resistencia que se genera ante la vulneración de derechos fundamentales de los cuales son titulares dichos asociados; la ineficacia de la norma se presenta pues, quienes están obligados a su cumplimiento, se niegan a hacerlo, porque no existe en ellos un reconocimiento de ese ordenamiento en tanto constituye un hecho atentatorio de sus derechos fundamentales.

“...El tema de los derechos fundamentales debe ser planteado de manera integral, para evitar que la atención exclusiva a uno de los derechos en juego nos haga perder de vista la complejidad del problema y la posibilidad de múltiples soluciones, en casos concretos." (Pavón Giraldo, 2008, pp. 82-83)

En consecuencia de todo lo planteado en este trabajo, y reafirmando lo que constituye uno de los puntos más importantes en el problema de investigación, 
resulta para nosotros probable que la ineficacia viene dada en gran parte por el escenario donde se han tomado las decisiones del aborto en Colombia, demostrándose que el actual y los que se han utilizado a la fecha, han sido inadecuados porque no existe integridad normativa suficiente que concentre los esfuerzos estatales e incluso ponen en tensión a la autoridades, quienes deben combatir el delito y al tiempo ser responsables de la efectividad de un derecho que conduce a una actuación similar a la que debe ser penalizada.

\section{REFERENCIAS BIBLIOGRÁFICAS}

\section{Bibliografía Referenciada:}

Ley 599 de 2000.

Sentencia de Constitucionalidad numero C-355 de 2006.

ELIAS, Norbert. (1998). La Civilización de los Padres y Otros ensayos. Grupo Editorial Norma. Barcelona

BERMUDEZ, Valdivia Violeta, (1998). La regulación jurídica del aborto en América Latina y el Caribe. Lima - Perú. 
VIVEROS, Mara. (1998), Problemas sensibles y estrategias metodológicas: A propósito de las investigaciones sobre aborto inducido. Centro de Investigaciones sobre dinámica social. Universidad Externado de Colombia.

\section{Bibliografía no referenciada:}

ARAUJO, Renteria, (2004). El derecho fundamental a la Igualdad: Precisiones. Bogotá. Derecho colombiano Ltda.

BALKIN, Jack M, (2005). Roe Vs. Wade, Should have said. USA. New York University Press.

BERMUDEZ, Valdivia Violeta, (1998). La regulación jurídica del aborto en América Latina y el Caribe. Lima - Perú.

BERNAL, PULIDO Carlos, (2005). El principio de proporcionalidad y los derechos fundamentales. Madrid - España. Centro de Estudios políticos y constitucionales. 
DWORKING, Ronald. El dominio de la vida: Una discusión acerca del aborto, la eutanasia y la libertad individual. Barcelona - España: Editorial Ariel S.A. 1991.

FAUNDES, Aníbal; BARZELATTO, José, (2005). El drama del aborto. En busca de un consenso. Colombia: Tercer mundo editores.

GALEANO Bejarano, Cristina Andrea, (2005). Intervencionismo de Estado en el control de natalidad en Colombia. Bogotá: Universidad Externado de Colombia - Tesis de Grado.

GLENDON, Mary Ann, (1987). Abortion and divorce in Western law. American failures, European challenges. USA: Harvard College- Harvard University Press.

KANT, Manuel, (2003). Fundamentación de la Metafísica de las costumbres. Crítica de la razón práctica. La paz perpetua. México: Porrúa.

KELSEN, Hans, (2001). Qué es Justicia?. Barcelona - España. Páginas 283: Editorial Ariel. 
LAURENZO Copello, Patricia, (1990). El aborto no punible. Barcelona - España. Bosh: Casa Editorial S.A. S.P.I.C.O.M.

O’NEILL, Onora, (1995). La Ética Kantiana. Madrid - España: Alianza editorial. 1995. OTERO Convers, Teresita, (1988). Aborto y legalización. Bogotá.

PEREZ Villa, Jorge, (2006). Constitución Política de Colombia - Comentada. Bogotá: Leyer Editores.

WARREN, Mary ann, (1995). El aborto. Madrid - España: Peter Singer (ed)Compendio de Ética - Alianza Editorial.

WARTEMBERG, Lucy, (1999). Embarazo precoz y aborto adolescente en Colombia. Bogotá: Centro de Investigaciones sobre dinámica social Universidad Externado de Colombia.

ZAMUDIO, Lucero, (1998). Problemas sensibles y estrategias metodológicas: a propósito de las investigaciones sobre aborto inducido. Bogotá: Centro de investigaciones sobre dinámica social - Universidad Externado de Colombia. 
ZAMUDIO, Lucero, (1999). El aborto inducido en Colombia. Bogotá: Centro de Investigaciones sobre dinámica social - Universidad Externado de Colombia.

ZAMUDIO, Lucero, (2000). El aborto en Colombia, dinámica socio demográfica y tensiones socioculturales. Bogotá: Universidad Externado de Colombia. 